\title{
Parametric design process basing on building physical performance
}

\author{
Yangluxi Li ${ }^{1}$, Lei Chen ${ }^{1}$ \\ ${ }^{1}$ Welsh School of Architecture, Cardiff University, UK
}

\begin{abstract}
The purpose of this research is to investigate general workflow of parametric architecture design basing on building physical performance. Earlier study on parametric design have shown that this method can effectively improve quality and diversity in terms of architecture appearance. In addition, design time could also be saved in this way. Nevertheless, research about parametric design on building configuration basing on building physical performance is still insufficient. Hence, this study investigates the specific workflow of parametric design in the basis of building performance. The result show that whole workflow can be divided into three steps:1) variables input 2) objective function determination 3) optimization operation. Combination of different component conducts in every step and realize final optimal scheme.
\end{abstract}

\section{Introduction}

This study focuses on two research areas: parametric design and building thermal environment performance. The purpose is to construct a parametric design process based on the building thermal physical environment combining above two areas. The optimized design of the sunshade components is described as a case.

Research done so far in the past ten years mainly concentrated upon intelligent optimization algorithms. For example, Caldas and Norford combined DOE2.1E with GA to optimize the geometrical variables of the office buildings façades with the annual total energy consumption as the objective function. Coley and Schuka also considered GA as the optimization algorithm and took the lowest building energy consumption as the objective function. ${ }^{[1-3]}$ In addition, the parametric model was also used to study the influence of thermal conductivity and heat capacity on the target function value. Meanwhile, the architecture subjective judgment was regarded as an optimal solution and the factors of manual intervention were added on the basis of algorithm calculation simultaneously. ${ }^{[4-5]}$ In this case, Trubiano et al. integrated software Radiance, Energy Plus and MATLAB with GA to optimize the shape of an office building with an atrium in terms of total energy consumption and indoor illumination. ${ }^{[6]}$

\section{Methodology}

\subsection{Object}

The research object of this paper is the parametric optimization devising method in accordance with building thermal performance. The key of parametric design is the collection of prototypes, adapting the prototype to the established goal of the design. In this process, the architect controls the archetype produced rules and sets variables - parameters. Moreover, morphology as a geometric representation is only the function of the parameter threshold in the threedimensional space under the generated rule. Therefore, the result of such operation is infinite rather than only a single solution. The architect utilizes and modifies the rules or re-substitute the parameters based on the feedback to pick up the most optimized scenarios.

\subsection{Process}

Traditional building performance optimization research often adopts the exhaustive method. The main problem of such methods is the huge time cost, especially in solving the practical problems of complex relationship between input and output. In addition, the accuracy and diversity of exhaustive optimization results also limit their development. With the increasing application of intelligent optimization algorithms in many fields such as artificial intelligence, system control, material distribution, pattern recognition, etc., it has significant potential in disposal of complexity, nonlinearity, multipole, large-scale and other issues. Researchers in the field are also beginning to pay attention to such algorithms and try to solve various problems in architectural design optimization.

Evolutionary algorithm has many advantages in the field of architectural design optimization in which genetic algorithm is the main algorithm. In view of the application process, not only can it be combined with the building performance simulation, also applied to the initial stage of the architectural design. In addition, the environmental monitoring data can also be integrated

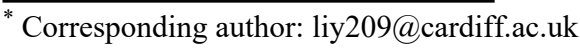


with algorithm to realize the intelligent regulation of the subjective system or variable construction components to achieve the optimization of building performance. The workflow of optimized algorithm with building performance is shown as follows Fig.1 and Fig.2.

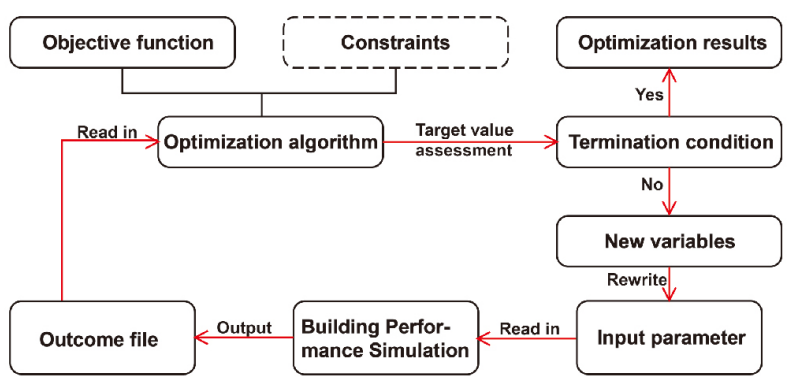

Fig.1. Workflow combining optimization algorithm and building performance simulation software (design stage)

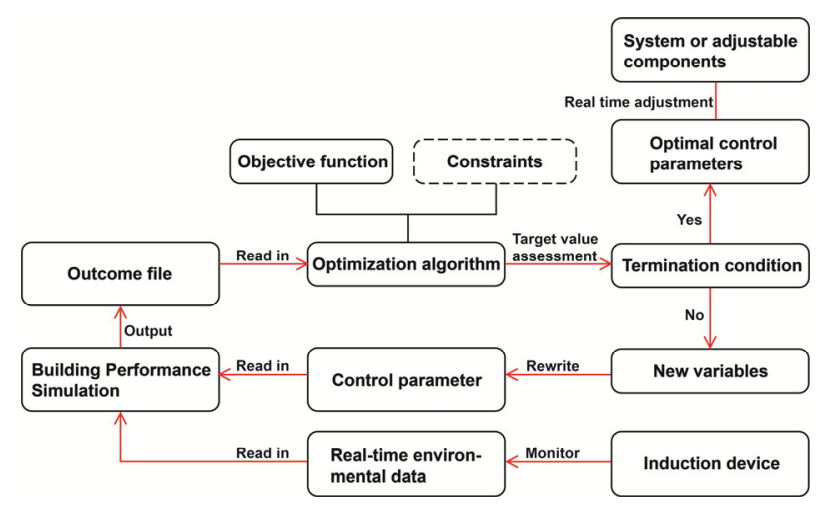

Fig.2. Workflow combining optimization algorithm and building performance simulation software (operation stage)

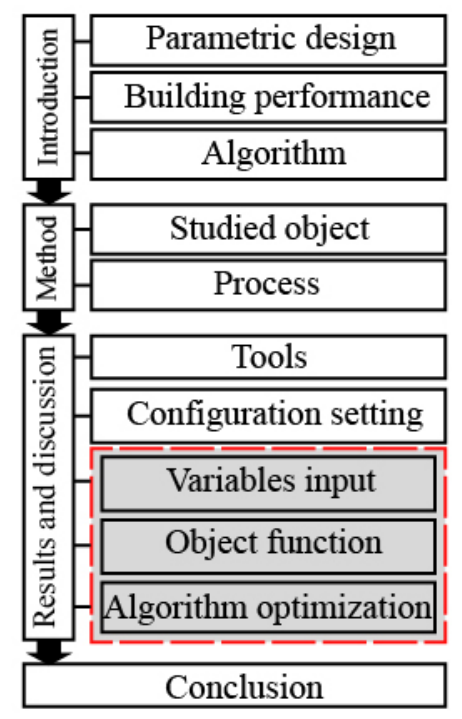

Fig.3. Research route of this study

The basic process of parametric design can be called "finding shape" which changes the geometric variable values of the prototype to generate a controllable building shape under a preset target or constraint. Since parametric design can create many architectural shapes beyond designer's expectations, the advantages of parametric architectural design means are summarized into three aspects as follows. 1) Parameterization. The morphological generation algorithm improves the designer's modelling ability and efficiency, and even forms a feedback about the design concept. 2) Multiformity. Variants inputting effectively increases the diversity of process outputs. 3) Function. The logical structure and function relationship between variants and construction pattern in design course considerably enhances the objectivity of design and also strengthens the difference for various schemes. Whole research process graph of this study is drawn as Fig 3.

\section{Results and discussion}

\subsection{Tools}

Table 1 parametric design tool

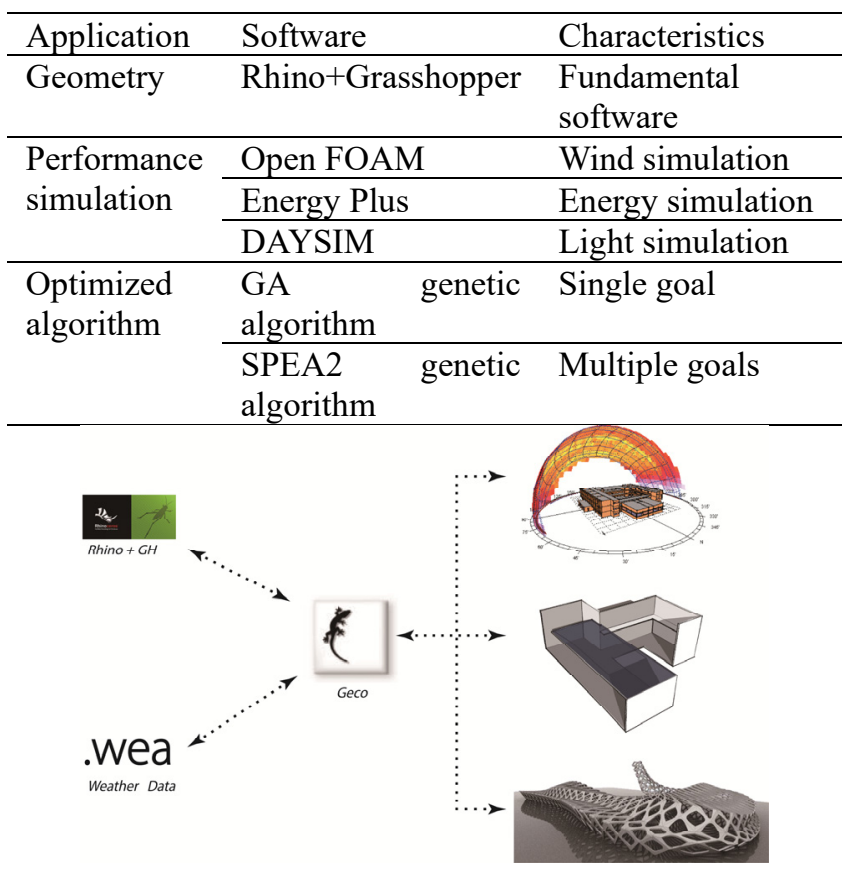

Fig.4. Relationship of simulated softwares

Table 1 indicates the common tools for parametric design. Rinosceros is considered as the main geometric software as its remarkable curve modelling function which parametric design mainly bases on. In addition, Rinosceros plug-in Grasshopper is the mainstream parametric programming platform which can integrate a variety of plug-ins to analyse climate, energy consumption and other nodes with other software in python programming language.

To study the parametric building design basing on building performance, data exchange must be carried out in the parametric platform and the building physical performance platform. Currently, relevant interface modules (plug-ins) are more commonly used in grasshopper, including Geco. Ladybug and Honeybee. Geco is the interface module between grasshopper and Ecotect. In this study, GECO is linked with real-time solar data. Ladybug and Honeybee are the interface modules of grasshopper and Energyplus.(Fig.4) 
Beyond above, another significant part of parametric optimization design is the genetic algorithms. At present, common optimization algorithms contain evolutionary algorithms, direct search methods and hybrid algorithms. Galapagos component (Fig. 5) is a genetic algorithm module embedded into Grasshopper. Genetic algorithm is an algorithm based on the survival principle of Darwin's biological evolution theory for adaptive global optimization. It can directly work on structural objects and the search space adaptively adjusts the direction with no need to determine the rules. In Grasshopper, the galapagos component is regarded as an automatic optimization process. It has two output nodes. The Genome node is associated with the argument parameters of the parametric model, and the Fitness node is linked with the dependent variable. When the Galapagos component operates, it automatically adjusts the parameter value of the independent variable, so that the dependent variable reaches a reasonable parameter, which can be a maximum or minimum value, or a custom value.

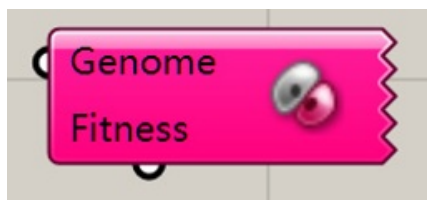

Fig.5. Galapagos component

\subsection{Parametric optimized design of special components of building facades as a case of sunshade components.}

Building exterior sun shading is one of the effective means of building energy efficiency. The outer sunshade components are supposed to be considered as maximum level in sun-protected on summer and hot in winter. As the external shading components affects building energy performance, it is more reasonable to define the total energy consumption as the goal function.

The case is assumed to be optimization for exterior sun shading component with the form of an external fixed horizontal sunshade louver in the south facade for a single office in a multi-story building. (Fig.6) The solar radiation data monitored is used as meteorological data. The initial variables include: the distance between the inner edge of the blade and the outer surface of the wall, the inclination of the blade, and the overall position of the blade. The objective function is total building energy consumption.

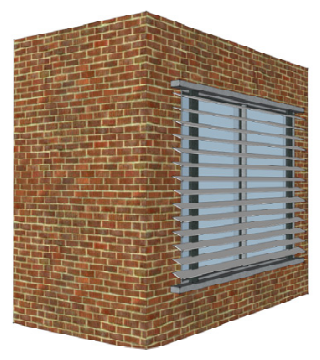

Fig.6. Case of office building with exterior sun shading component

\subsection{Configuration setting}

\subsubsection{Geometrical Variables}

1.As the grasshopper plugin only could recognize HBSurface model format, it is imperative to convert the geometric surface representing the office to HBSurfaces using the createHBSrfs node.

2.Adding a window surface to the south facing wall using the addHBGlz components as follow Fig. 7.

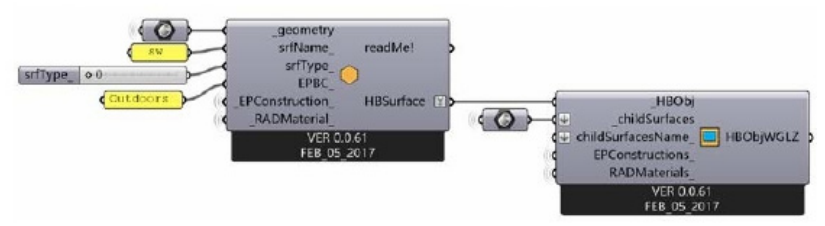

Fig.7. Geometrical variable components

3.Create an HB partition. Create an HB partition with the createHBZones node preparing for energy calculation.

4.Create sunshade components. Using the Window Shade Generator node, input the HBObj output from above third step into the HBObjects node, and enter 0 into the shadeType node to indicate that the sunshade component type is louver; set the corresponding Slider and enter the distToGlass, depth, numOfShds, and shdAngle interfaces respectively representing the detail information of sunshade components.

\subsubsection{Material variables}

In order to reduce calculation time, the simplification method can be used to create the enclosing material in which the multi-layer material of the opaque enclosing structure is assumed to be a single material. Furthermore, the real material has the same total thermal resistance as the multi-layer material. Create architectural opaque materials with the EnergyPlus NoMass Opaque Material node. Create EP opaque envelope construction using EnergyPlus Constrcution node. Create transparency material and construction of windows. Creat EP and RAD sun shading material using Energyplus shade material node. In addition, setting EP and RAD material for envelop construction via inputting above material parameters into createHBSrfs 、addHBGlz、EP context Surfaces nodes.

\subsubsection{Building operation variables}

Set EnergyPlus Zone Loads node for office calculating energy consumption and then use the Annual Schedule node to generate staff working rates and equipment running schedules.

\subsubsection{Calculate total energy consumption}

Input the resultFileAddress which is output from the Run Energy Simulation node to the resultFileAddress in Read EP Resultcomponent. Basing on the Separate Data 
component, input the cooling, heating, electric Light, and electric Equip output from the Read EP Result node into the input List interface in turn. As for the total energy consumption, this value is got via summation from all values above number nodes.

\subsubsection{Optimization}

In the optimized process, related parameter can be set in the octopus component and input variants in Optimize Preferences panel. General reference parameters can be used as follows. Elitism: 0.70; Mut. Probability: 0.050; Mutation Rate: 0.3; Crossover Rate: 1.0; Population Size: 30; Max Generation: 20. Pareto convergence (SPEA-2) and polynomial mutation algorithm (Polynomial Mutate) is considered as the convergency method and algorithm. Finally, according to the optimized computed result, algorithm calculates the recommended variables under the lowest energy consumption. Figure 8 shows the optimized results via algorithm computation.

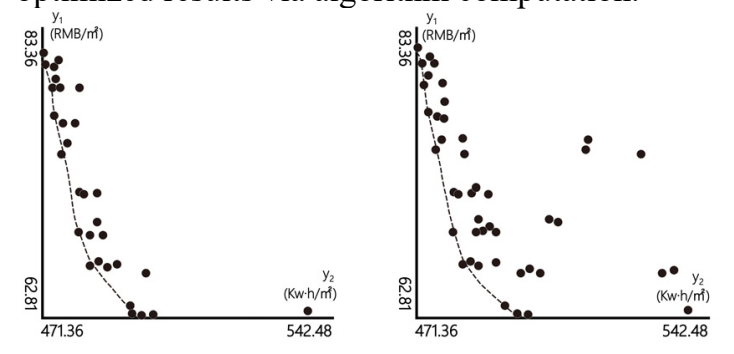

Fig.8. Optimized results via algorithm computation

\section{Conclusion}

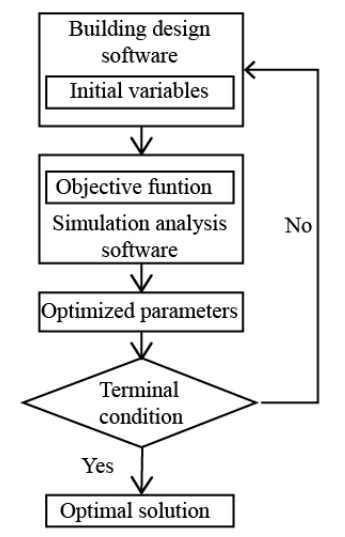

Fig.9. Whole workflow parametric design based on building performance

According to above analysis, parametric design workflow can be built basing on building performances. Initial variables, goal function and optimized parameter are of significance for process. (Fig.9)

Initial variable. The initial variable refers to the value that the architect can directly set, modify, delete or add in the process of design optimization. The initial variables dominate various aspects of the building shape, including building group geometric variables, single building geometry variables, building material physical variables, price variables and other variables. The building objective function actually is the value of the initial variable under a specific condition, so the optimization of objective function is essentially the process of adjusting the initial variable. Note that a particular objective function may be affected by multiple initial variables, but usually has some primarily affected initial variables.

Objective function. The objective function refers to the concrete manifestation of the architectural performance optimization design goal. It is the mandatory constraint condition for the optimization of the building performance and plays a remarkable role in judging whether the performance of the design scheme meets the requirements. Architects designate the objective function required in line with various specifications and standards. In fact, there may be multiple objective functions to perform for the same devised scheme. Architect should conduct the appropriate objective function based on the actual requirement of the project and the difficulty of calculation, measurement and evaluation.

Optimized algorithm. The optimized parameter controls the corresponded algorithm to perform the calculation. Such parameters have a significant impact on the speed, accuracy, and convergence of the optimization process. For genetic algorithm (GA), for example, four optimization parameters i.e. population size, termination evolution algebra, crossover probability and mutation probability need to be determined before the algorithm running. Therefore, above relative corresponding parameters such as max. total, inbreeding, and mutations are also required when using the genetic algorithm module Galapagos in Grasshopper. Note that although the optimization parameters have a significant influence on the optimized results, there is still no reasonable and uniform selection basis at present. Determining the reasonable value of such parameter generally utilizes trial calculation mean.

As shown in figure, architect directly builds the building model in software first. Meanwhile, this process also assigns and manipulates the initial variables. Then, performance simulated software obtains objective function value via extracting the initial variables in the building design software under the specific calculation rules. Finally, the optimization algorithm tool evaluates the fitness degree for diversified schemes in the basis on the objective function value, thereby continuing or terminating the optimization process (simulation result application).

\section{References}

1. W. Richardson, H. Krishnaswami, R. Vega, M. Cervantes, Sustainability, 9(2017)

2. L. Yang, X. D. Liu, F. Qian, J. Clean Prod.209(2019)

3. R. Marquez, C. F. M. Coimbra, Solar Energy, 91(2013)

4. Y.L.X. Li, Proceedings of 2016 International Conference on Modeling, Simulation and Optimization Technologies and Applications. (2016)

5. M. Wetter, J. of Built Performance. 2(2009) 
6. D. Hu, P. Jones, E. L. Segarra, C. F. Bandera, 4th Building Simulation and Optimization Conference, Cambridge, UK(2018) 